\title{
The Design of Electrical Equipment Overheat Monitoring System on Electric Propulsion Ship Based on WSN
}

\author{
Ma Chuan ${ }^{1,2, a}$, li Diyang ${ }^{1}$ and Mao Hongyu ${ }^{1}$ \\ ${ }^{1}$ Engineering Department, Qing Dao Ocean shipping Mariners College, Qing Dao, China \\ ${ }^{2}$ Engineering Department, Da Lian Maritime University, Da Lian, China
}

\begin{abstract}
Wired electric equipment overheating monitoring system has some limitations in electric propulsion ships. This paper designs an electrical equipment overheat monitoring system based on Wireless Sensor Network (WSN) to monitor the overheat of electrical equipment. To verify the performance of the whole system, we design the hardware and the software of sensor nodes and coordinators. In order to extend the lifetime of sensor node effectively, the system adopts the dynamic sleep and controllable transmit power method. The results show that this system achieves the accurate measurement of electrical equipment temperature. At the same time, laboratory data tests shown that it can achieve the same accuracy as wired measurements. This paper provides a new solution for overheating monitoring of marine electrical equipment.
\end{abstract}

\section{Introduction}

Wireless Sensor Network (WSN) is composed of a large number of tiny sensor nodes with communication and computing capabilities. At present, it is widely used in military, industrial, medical, agricultural, environmental monitoring, smart cities and other fields ${ }^{[1]}{ }^{[2]}$. The electric propulsion ship has the advantages of good maneuverability and economy, little vibration and noise, flexible cabin space layout etc. ${ }^{[3]}$, which has become the development direction of modern shipbuilding. Electric propulsion ships have much important electrical equipment, real-time monitoring of the superheat of these electric equipment helps the engineer to predict faults in advance and take measures to avoid the expansion of faults.

Recently, the monitoring of the electrical equipment temperature on electric propulsion ships generally adopts the traditional wired temperature measurement and infrared temperature measurement methods. Wired temperature measurement method requires a large number of cables to be laid, and it is difficult to achieve high voltage isolation. Although infrared temperature measurement method can achieve high voltage isolation, it requires staff to periodically patrol temperature measurement, which cannot guarantee real-time measurement and accuracy is not high. At the same time, the accuracy maybe affected by the environment.

For the above problems, this paper introduces WSN technology into marine applications, and develops a WSN-based electric propulsion ship electrical equipment temperature monitoring system, which can realize realtime accurate temperature measurement, high voltage isolation and overcome the limitation of traditional method. This becomes the development trends of medium and high voltage electrical equipment monitoring.

\section{2 system architecture}

The WSN-based electric propulsion ship electrical equipment monitoring system is composed of sensor nodes, routing nodes, central nodes, MODBUS communication units, and computer application software (as shown in Figure 1).

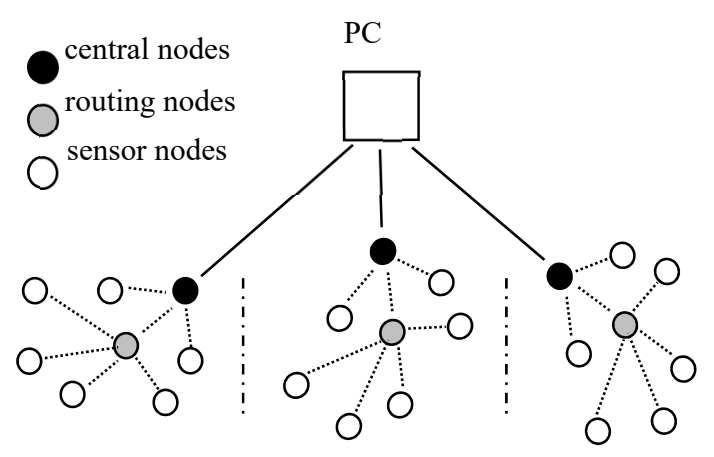

Figure 1. System architecture.

The sensor node is mainly composed of digital temperature sensor, CPU, and a power module. In the case where wiring is inconvenient, the sensor node is powered by lithium battery. Its main function is to collect the temperature of monitoring point and automatically enter the sleep state according to the temperature change to achieve energy saving. The main function of the routing node is to route the data transmitted by the sensor node. If the routing node's next hop routing address is central node, the data is passed to the end point. If the routing node's

\footnotetext{
a Corresponding author: machuan1984@126.com
} 
next hop routing address is still a routing node, the router will pass the data to the next router to continue routing the data. The central node is mainly responsible for temporarily storing data and waiting for computer to read. In addition, the central node is also responsible for maintaining the WSN, receiving the command information of the upper computer and changing the parameters of the network. The upper computer application software is designed by NI LabVIEW software, and its main function is to process the data sent by the central node.

\section{Hardware design and implementation}

The hardware design(as shown in Figure 2) of this system is different from the traditional monitoring system. Since some of the WSN sensor nodes work in the state of lithium battery power supply ${ }^{[4]}$, it is required to consider energy saving in hardware design. At the same time, in electric propulsion ships, there are much electrical equipment and $6.6 \mathrm{KV}$ medium voltage equipment, so high voltage isolation and electromagnetic interference should be considered in hardware design.

The microprocessors of central node and sensor nodes are all selected TI's CC2530. This chip is superior in performance and cost to the same type of chip. At the same time, TI also customized a completely free protocol stack for the CC2530, making the design of the program more convenient. The chip integrates a $2.4 \mathrm{GHz}$ wireless transceiver conforming to IEEE 802.15.4 standard. The RF unit has very high receiving sensitivity and strong anti-interference performance. In sleep mode, only 0.9 microamps of current is required, and the energy saving performance is very good. Suitable for battery powered devices.

The sensor node is powered by lithium battery, so when designing the circuit, it must ensure that the node can turn off the sensor power supply during sleep to save energy. The circuit only works during the operation of the sensor, and the rest of the time is in the power-off state. The sensor node uses the digital temperature sensor DS18B20, which directly converts the temperature into a digital signal and sends it to the CPU.

In addition, the sensor node works in high-voltage electric field and various interferences. Therefore, the system is designed with full consideration of the electric field shielding, a shield is added to the sensor node and the shielding layer is reliably grounded. All chip input pins are pulled up by resistors to prevent the chip from being disturbed by external electric fields as little as possible. When designing the $\mathrm{PCB}$, the ground line is thickened and the board is covered with copper over a large area to reduce the interference caused by the ground line. The node was also designed with the "watchdog" feature.
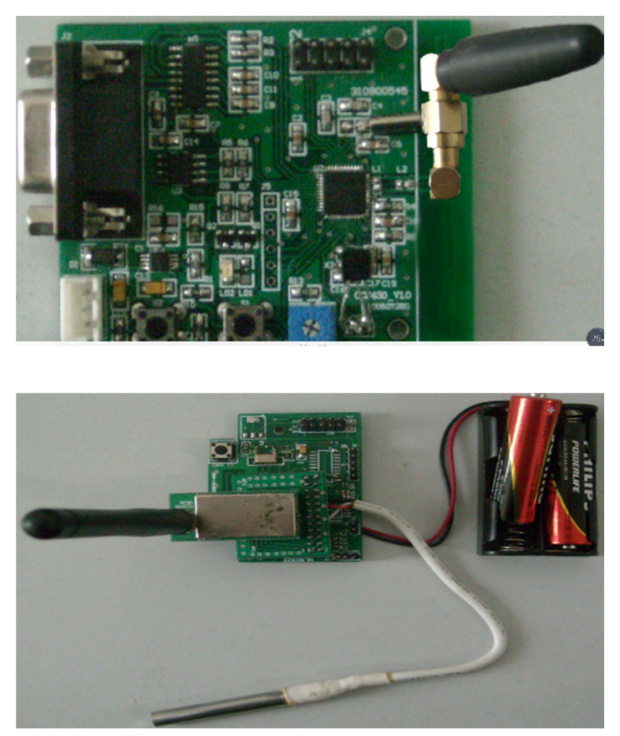

Figure 2. The actual photo of central node and sensor node

\section{Software design and implementation}

The software and hardware of the system complement each other to realize the functions of the system. The overall structure of system software is divided into three layers: the software of the sensor node (temperature acquisition, transmission power control, command processing), the software of the central node (MODBUS communication software, command processing software) and the application software of the computer.

\subsection{Software design of central node and sensor node}

After the central node is powered on, a new WSN network is first formed. After the network is formed, the application layer program is executed to initialize the user program. After initialization, the program will continuously detect if data is entering. When the central node receives the data sent by the communication module or the wireless data sent by the sensor node, it will enter the receiving program. If the data comes from the host computer, enter the command processing subroutine to analyse and process the data. If it comes from the sensor node, enter the temperature processing subroutine.

After the sensor node is powered on, it first searches for the wireless sensor network in the area. If it searches for the network and successfully joins, it starts executing the application and starts collecting the temperature. After collecting $\mathrm{M}$ times, the average is sent to the central node. At the same time, the rate of temperature change is calculated. According to the changing rate, the node calculates the sleep time and put the sensor node into sleep state to achieve maximum energy saving. After the sleep time is reached, then $M$ temperature collection is performed.

\subsection{PC application software design}


The computer application software is designed by NI LabVIEW software. The software interface has the characteristics of simplicity and can intuitively display the information of each monitoring point in front of the management personnel. In the design of the host computer interface, the display picture is matched with the actual electrical equipment as much as possible, so that the engineer can locate the temperature of the electrical equipment monitoring point in a short time as shown in the figure. The application has the following functions:

(1) Display and alarm function of monitoring point parameters.

(2) Timed printing and summoning printing of parameters.

(3) Blocking of parameter alarms and communication parameter setting function.

(4) The parameter overrun fault prediction function.

\section{Sensor node energy saving control}

Part of the sensor nodes in the system use battery powered, and energy-saving control of this type of node is necessary. The transmitting energy of the RF transmitter is proportional to the transmission distance. The ideal energy-saving scheme is to change the transmitting power of the transmitter as the transmission distance changes, thereby achieving energy saving. When the distance between nodes is constant (as shown in Figure 3), the distance between node $\mathrm{A}$ and node $\mathrm{B}$ is $\mathrm{d}$. When $\mathrm{A}$ and $\mathrm{B}$ communicate, data is transmitted at a transmission power of $10 \mathrm{mw}$, and Node $\mathrm{B}$ can receive data safely and reliably. If node A transmits data at a power of $20 \mathrm{mw}$, node $\mathrm{D}$ also transmits data at a power of $20 \mathrm{mw}$, node $\mathrm{B}$ can receive data of node $\mathrm{A}$, and node $\mathrm{C}$ can also receive data of node D. However, the coverage range transmitted by Node A overlaps with the coverage range transmitted by Node D, which causes collisions between channels. In the case of numerous nodes, data may be lost ${ }^{[5][6]}$. More importantly, after the transmission power is increased, unnecessary energy waste is caused.

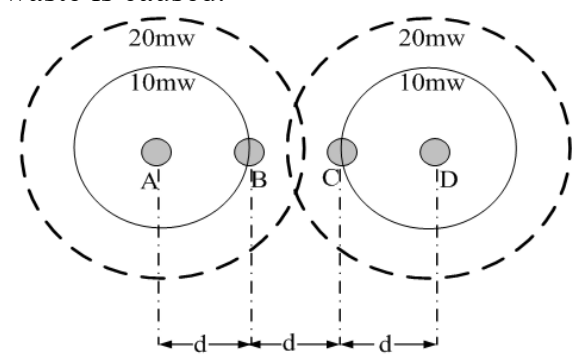

Figure 3. The range of transmitting power between nodes

According to the transmission formula of radio waves in free space, namely the Friris transmission formula ${ }^{[7][8]}$ :

$$
\mathrm{P}_{\mathrm{r}}=\frac{\operatorname{Pt} \mathrm{GtG}_{\mathrm{r}}}{\mathrm{L}}\left(\frac{\lambda}{4 \pi \mathrm{d}}\right)^{\mathrm{n}}
$$

Where $G_{t}$ and $G_{r}$ are the gains of the transmitting and receiving antennas, $\lambda$ is the carrier wavelength, $d$ is the distance between transmission and reception, and $\mathrm{L}$ is the loss factor. When $G_{t}, G_{r}, \lambda$, $d$, and $L$ are all determined, $P_{r}$ can be obtained from the equation as long as $\mathrm{P}_{\mathrm{t}}$ is known. From equation 4.1 can be obtained that:

$$
\mathrm{P}_{\mathrm{m}}=\frac{\mathrm{L} \mathrm{P}_{\text {Thresh }}}{\mathrm{GtG}_{\mathrm{r}}}\left(\frac{4 \pi \mathrm{d}}{\lambda}\right)^{\mathrm{n}}
$$

Combine formula 1 with 2 can be obtained:

$$
\mathrm{P}_{\mathrm{m}}=\frac{\mathrm{Pt}_{\mathrm{P}} \mathrm{P}_{\text {Thesh }}}{\mathrm{P}_{\mathrm{r}}}
$$

Where $\mathrm{P}_{\mathrm{m}}$ is the best transmit power, $\mathrm{P}_{\text {Thresh }}$ is the receive threshold power, $\mathrm{P}_{\mathrm{t}}$ is the transmit power, and $\mathrm{P}_{\mathrm{r}}$ is the receive power. According to (3), it can be known that the value of the optimum transmission power $\mathrm{P}_{\mathrm{m}}$ can be obtained by knowing the threshold power $\mathrm{P}_{\text {Thresh }}$, the transmission power $\mathrm{P}_{t}$ and the reception power $\mathrm{P}_{\mathrm{r}}$ of the receiver. The value of $\mathrm{P}_{\text {Thresh }}$ is $-91 \mathrm{dbm}$ of CC2530. In addition, the received power value $\mathrm{P}_{\mathrm{r}}$ can be obtained by the RSSI VAL bit in the register RSSIL of the chip, so that the transmit power value $\mathrm{P}_{\mathrm{t}}$ can be known and then the value of the optimal transmit power $\mathrm{P}_{\mathrm{m}}$ can be calculated according to Equation (3) ${ }^{[9][10]}$. The flow chart of the scheme for obtaining the optimal transmit power of the sensor node designed by the system is shown in Figure 4 and Figure 5.
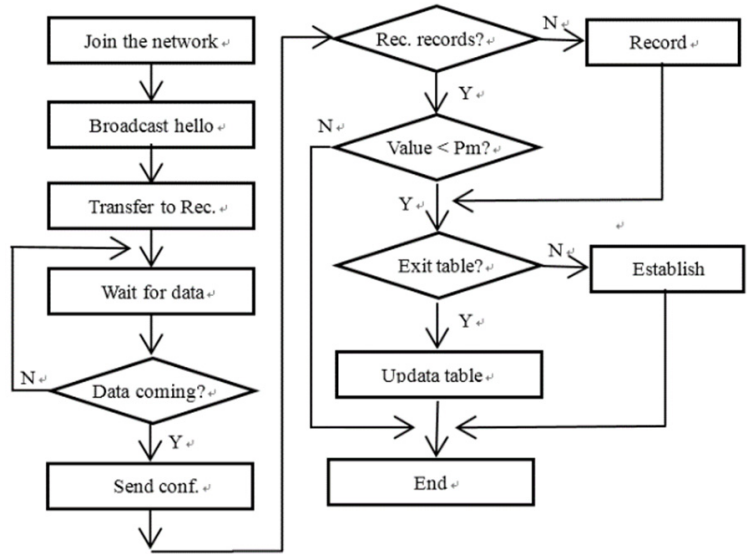

Figure 4. The flow of sensor nodes

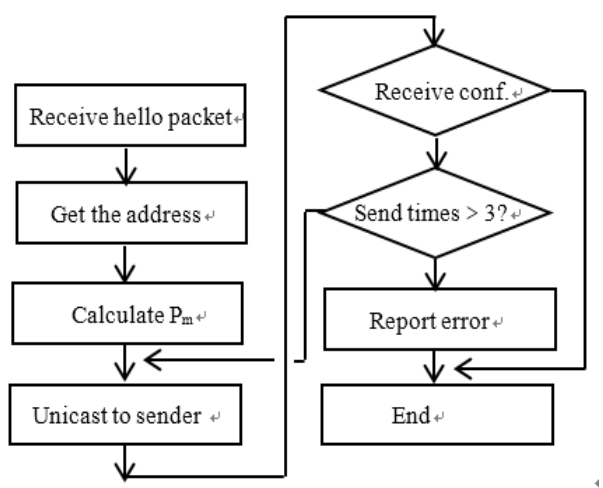

Figure 5. The flow of central node

\section{6 system performance verification}

In order to verify the performance of the WSN-based electric propulsion ship electrical equipment monitoring system designed in this paper, the performance of wireless sensor network nodes and wired temperature acquisition are compared under laboratory conditions. The specific implementation is as follows: 
(1) The sensor node with dynamic sleep designed in this paper and the wired temperature measurement node are simultaneously placed in the same container in the laboratory.

(2) The laboratory container is heated by electric heating to gradually increase the temperature from room temperature to $100{ }^{\circ} \mathrm{C}$.

(3) Wired temperature measurement adopts $200 \mathrm{~ms}$ interval sampling. The sensor nodes designed in this paper adopt dynamic sleep mode for acquisition (according to the scheme designed in this paper, the acquisition interval is dynamically adjusted according to the temperature change rate).

(4) Put the results of the two in the same chart, observe the difference between the collection results of the sensor nodes with dynamic dormancy and the wired acquisition results.

(5) The results show that the WSN-based electric propulsion ship electrical equipment monitoring system and the wired temperature monitoring system have the same high measurement accuracy.

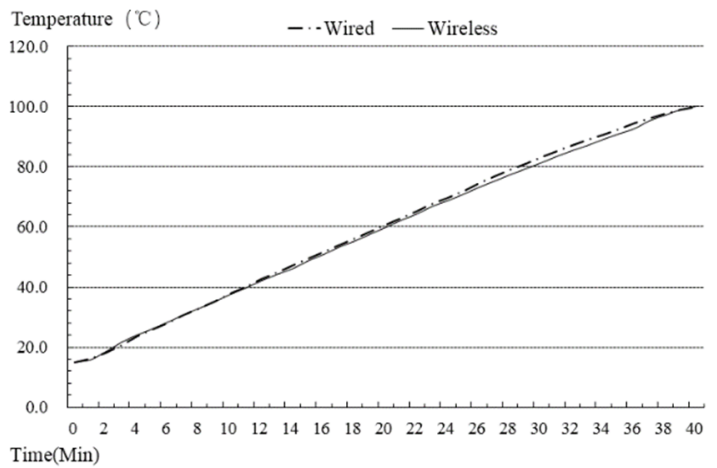

Figure 6. The comparation of wired and wireless temperature measurement.

\section{Conclusion}

In this paper, a wireless electric equipment overheats monitoring system based on WSN is proposed. The remarkable feature of this system is that compared to wired system, it is convenient to realize temperature measurement and wireless low-power transmission of temperature data. When the temperature data of the monitoring point is abnormal, an alarm will be given to remind the engineer to handle it. The design of this system can greatly improve the operational reliability of electric propulsion ship electrical equipment. However, the content of electric propulsion ship electrical equipment monitoring is extensive and complex. The overheating monitoring of electrical equipment developed in this paper is only one of them. The design of this system provides a theoretical basis and practical significance for the realization of smart cabin for electric propulsion ships.

\section{Acknowledgements}

This paper was financially supported by COSCO Shipping Group Research Foundation under Contract No. 2018-1-H-016.

\section{References}

1. R.J. Aarti, K. Karishma, International Research Journal of Engineering and Technology. 4,1371-1378 (2017)

2. M. Aliyu, A. Abdullah, H. Chiari, Int. J. Distrib. Sens. Netw. 3,1-9 (2016)

3. X. Gu, J. Yu, G. Wang, Comput. Electr. Eng. 40,384398 (2014)

4. B. Gurakan, O. Ozel, J. Yang, S. Ulukus, IEEE Transactions on Communications 61,4884-4898 (2013)

5. X.H. Liu, Wuhan University of Technology,5,1-120 (2010)

6. R. Ma, Y.C. Liu, C. Wang, Journal of Dalian Maritime University,40,77-80 (2014)

7. A.R. Raafat, N.K. Al-Ali, Industrial Informatics and Computer Systems, International Conference on. IEEE,1,1-5 (2016)

8. J.C. Xie, Ship Engineering 2,61-64 (2011)

9. Z.W. Zeng, Z.G. Chen, A.F. Liu, Chinese Journal of Computers 33,12-22 (2010)

10. L. Zheng, Industrial wireless sensor networks and standardizations, 1,1187-1190 (2010) 